\title{
Observer techniques for estimating the State-of- Charge and State-of-Health of VRLABs for Hybrid Electric Vehicles
}

\author{
B. S. Bhangu (MIEEE), P. Bentley, D. A. Stone, C. M. Bingham (MIEEE) \\ Department of Electronic and Electrical Engineering \\ University of Sheffield, Mappin Street, \\ Sheffield, S1 3JD, UK. \\ Corresponding author: b.bhangu@sheffield.ac.uk
}

\begin{abstract}
The paper describes the application of observerbased state-estimation techniques for the real-time prediction of state-of-charge (SoC) and state-of-health $(\mathrm{SoH})$ of lead-acid cells. Specifically, an approach based on the well-known Kalman Filter, is employed, to estimate SoC, and the subsequent use of the EKF to accommodate model non-linearities to predict battery SoH. The underlying dynamic behaviour of each cell is based on a generic Randles' equivalent circuit comprising of twocapacitors (bulk and surface) and three resistors, (terminal, transfer and self-discharging). The presented techniques are shown to correct for offset, drift and long-term state divergence-an unfortunate feature of employing stand-alone models and more traditional coulomb-counting techniques. Measurements using real-time road data are used to compare the performance of conventional integration-based methods for estimating SoC, with those predicted from the presented state estimation schemes. Results show that the proposed methodologies are superior with SoC being estimated to be within $1 \%$ of measured. Moreover, by accounting for the nonlinearities present within the dynamic cell model, the application of an EKF is shown to provide verifiable indications of SoH of the cell pack.
\end{abstract}

Index Terms - Batteries, Energy management, Energy storage Nonlinear estimation, State estimation.

\section{INTRODUCTION}

$\mathrm{C}$ ells employed in hybrid electric vehicles (HEVs) as peak power buffers operate under arduous conditions, being subjected to both large dynamic transients in current and power demand over a wide temperature range. Moreover, the cells are mostly operated at a Partial State-of-Charge (PSoC) i.e. the cell is never cycled over its full $\mathrm{SoC}$ range. An example is given by the RHOLAB driving cycle requirements, which shows road data collected from a Honda Insight HEV, Fig. 1, where peak charge- and discharge-current demands of $\approx 60 \mathrm{~A}$ and $\approx 100 \mathrm{~A}$, respectively, are required from the battery pack, Fig. 2 [1].

The accommodation of such operating conditions requires that the management system has accurate knowledge of the peak power buffer's SoC to facilitate safe and efficient utilisation of the battery for HEVs, by preventing under- or over-charging conditions, thereby extending the lifetime and preventing progressive permanent damage to the battery. Moreover, failure to control $\mathrm{SoC}$ can also degrade the ability of the pack to source/sink subsequent power transients.

An assortment of techniques have previously been reported to measure or monitor the SoC of a cell or battery, each having its relative merits, as reviewed by Piller et al [2]. Charge counting or current integration techniques, are, at present, the most commonly used strategy, requiring measurement of the cell/battery current, the time-integral of which is considered to provide a direct indication of SoC [3]. However, due to the reliance on integration, errors in terminal measurements due to noise, resolution and rounding are cumulative, and large SoC errors can result. A reset or recalibration action is therefore required at regular intervalsin electric vehicles (EVs) this may be carried out during a full charge or conditioning discharge, but is less appropriate for standard HEV operation where full SoC is rarely achieved. Other factors that ultimately influence the accuracy of SoC estimates, and create additional complications for the traditional integration-based techniques, are the variation of cell capacity with discharge rate, temperature and Coulombic efficiency losses.

When considering flooded lead-acid cells, the specific gravity of the electrolyte is known to be a good measure of SoC. However, estimates of SoC are complicated when using valve regulated lead-acid (VRLA) cells due to the nominal amount of electrolyte being immobilised in the glass fiber separator mat or gel. Nevertheless, since the open-circuit terminal voltage of a VRLA battery varies almost linearly over the majority of the battery's SoC [4], it has been used in many SoC estimators. For the method to be effective, however, corrections must be made for temperature and electrolyte concentration gradients (long settling times may be required to allow such concentration gradients to disperse prior to making an open circuit voltage reading [4]). More 


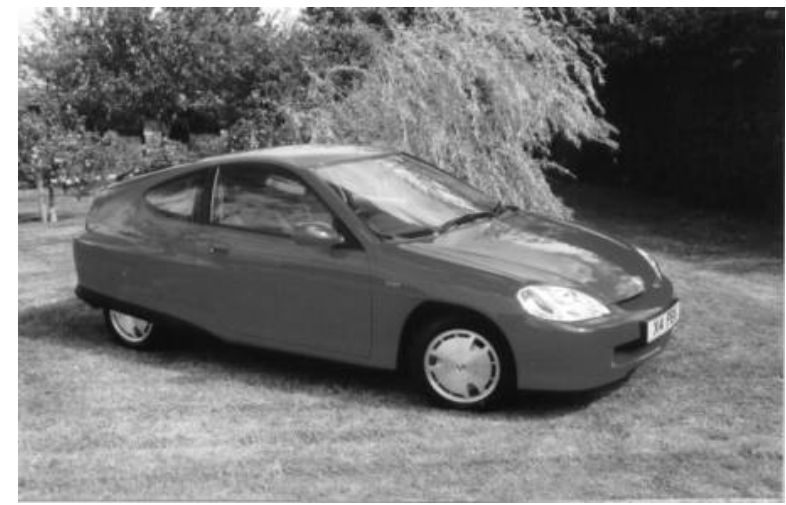

Fig. 1. RHOLAB Honda Insight HEV employed to gather test data.

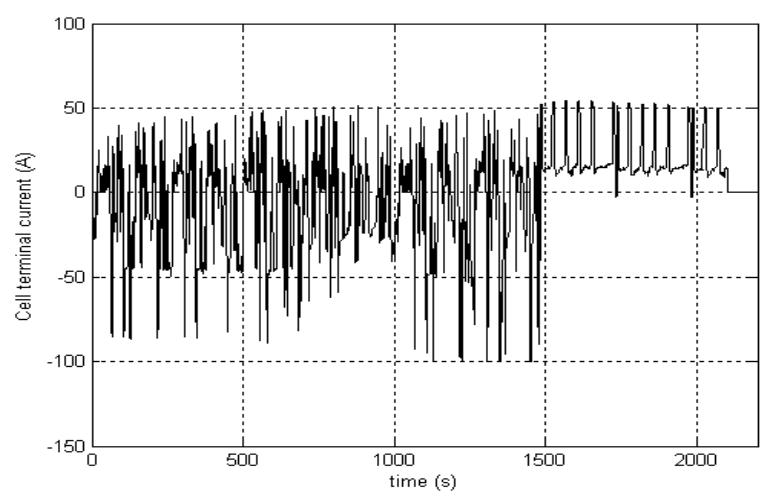

Fig. 2. Typical RHOLAB [1] driving cycle cell current.

commonly therefore, the measured open-circuit voltage is used to periodically correct the estimated $\mathrm{SoC}$ derived from other charge integration techniques. However, as with measurements of specific gravity, suitable periods of operational inactivity may not occur frequently enough in HEV driving duties for this to be successfully utilised.

Cell impedance measurements have also been reported as a useful technique for resetting or adjusting SoC estimates from integration-based methods. However, from results of various studies undertaken to identify the impedance variation of cells/batteries, with SoC [5,6], contradictory views to their usefulness in practical systems currently remain unresolved [2].

Other reported methods for estimating $\mathrm{SoC}$ have been based on artificial neural networks [7] and fuzzy logic [8] principles, although the latter was reported to have relative poor performance. Although such techniques incur large computation overhead on the battery pack controller, which would in the past have led to problems for online implementation, the increasing computational power of digital signal processing chips, and the accompanying fall in device costs may, in the near future, make their application an attractive alternative. Neural networks, in particular, have been used to avoid the need for the large number of empirically derived parameters required by other methods $[9,10]$. Indeed, for application to the less demanding task of prediction of $\mathrm{SoC}$ in portable equipment, a neural network modelling approach has been shown to give mean errors of $\approx 3 \%$ [9]. Also, a neural network model for predicting battery power capacity during driving cycles has been added to the ADVISOR EV and HEV modelling environments [10].

Various electric equivalent circuit models have been applied to lead-acid batteries to determine $\mathrm{SoC}$ [11]. However, the complex nonlinear electro-chemical processes that occur during power transfer to/from the battery has, in the past, proven difficult to accurately describe dynamically. These processes include the flow of ions, amount of stored charge, ability to deliver instantaneous power and the effects of temperature and internal pressure, to name a few. Although, in theory, the SoC of a battery can be determined from terminal quantities in conjunction with an appropriate battery model, inaccuracies and measurement noise ultimately introduce errors that can become significant over time.

Here then, an alternative technique is proposed for predicting the states of a cell that would normally be difficult or expensive to measure, or are subject to the significant problems, as described previously - the SoC being the key state in this case. Such model-based state-estimation techniques employ an error-correction mechanism to provide real-time predictions of SoC. Specifically, the well-known Kalman Filter (KF), developed during the 1960's to provide a recursive solution to optimal linear filtering for both state observation and prediction problems $[12,13,14]$, is used for this study; a unique feature of the KF being that it optimally (minimum variance) estimates states affected by broad-band noise contained within the system bandwidth i.e. that cannot otherwise be filtered out using classical techniques, and enables empirical trade-offs between modelling errors and the influence of noise.

Finally, manufacturers of HEVs would like predictions of the State-of-Health $(\mathrm{SoH})$ or State-of-Function $(\mathrm{SoF})$ of a battery pack, since the increasing reliance on drive-by-wire technologies is making the battery a key safety-critical component of the vehicle. Knowledge of whether a battery will fail when subject to high transient loadings, as may be experienced in emergency braking, for instance, is therefore essential. However, SoH monitoring techniques are currently in their infancy, with little being reported to-date. The proposed technique is therefore extended to accommodate estimation of SoH. Due to the resulting nonlinearities of the underlying dynamic model, an EKF is considered for real-time estimation of $\mathrm{SoH}$.

The cell tested in this paper is a novel, spiral wound, $8 \mathrm{Ah}$ sealed lead-acid cell, with terminals on either end, developed by Hawker (ENERSYS Inc), for the RHOLAB project, Fig. 3. The double terminal encapsulation is introduced to lower grid currents, and hence, thermal gradients, and thereby promote efficient utilisation of the active materials in the cell, thus leading to a battery that is optimised for the high-power duties typical of hybrid driving cycles. 


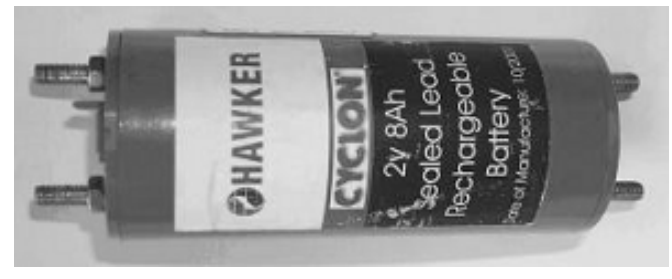

Fig. 3. 8Ah sealed VRLA test cell.

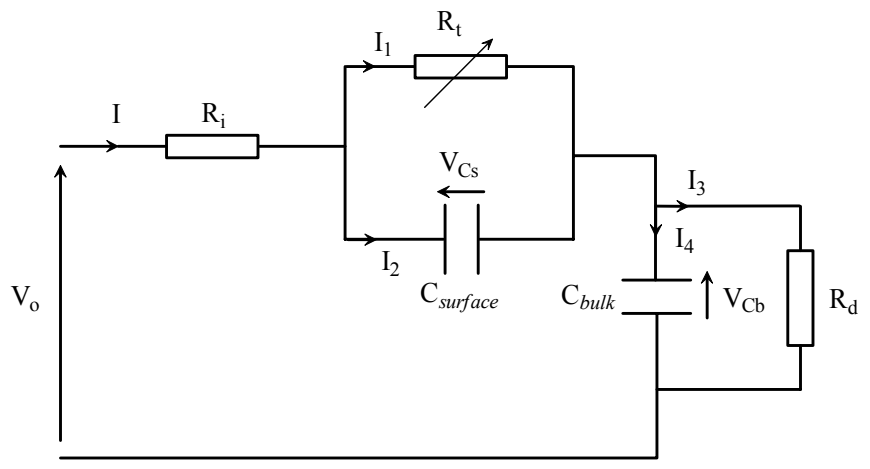

Fig. 4. Randles' type equivalent circuit of a lead acid cell [11].

\section{LEAD ACID BATTERY MODEL}

\section{A. Dynamic modeling}

A dynamic model of the lead acid cell, Fig 4, shows a simplified equivalent circuit accredited to Randles [16]. A model of this form is employed in union with an observerbased technique to simulate the response of VRLA cells to constant current and dynamic power cycles. The parameters of the simple equivalent circuit featuring lumped parameters for the two plates, are estimated using the voltage response and curve fits to data collected during current pulses applied to the VRLA double terminal cells, and is described in Sect. II-B. The cell terminal voltage is represented by $V_{o}$, and $R_{i}$ is a lumped resistance due to cell interconnections etc. A double layer capacitance $C_{\text {surface }}$ is shown in parallel with the charge transfer polarisation represented by $R_{t}$. This double layer capacitor is the result of charge separation at the electrolyte/electrode interface [15]. A charge double layer exists at both electrodes of the cell, and there may be more than one species of ion or molecular dipole contributing to its creation. In Fig. 4, $C_{\text {surface }}$ represents a lumped capacitance parameter for the interfaces at both the cell plates. Furthermore, $C_{b u l k}$ models the cell's open circuit voltage, and $R_{d}$ is included to represent the self-discharge of the cell. The voltages across the bulk- and surface- capacitors are denoted $V_{C b}$ and $V_{C s}$, respectively.

\section{B. Cell Parameter Characterization}

Impedance techniques provide a means of obtaining the parameters of the equivalent circuit, an example being shown in Fig. 5. The impedance is commonly measured using an applied excitation signal at the frequencies of interest $[15,16]$. Impedance spectroscopy has been used to identify electrochemical parameters for complex models of electric vehicle batteries [11,17], such as the double layer capacitance, charge transfer resistance and Warburg impedances. The application of impedance spectroscopy to large industrial batteries at low frequencies is discussed in [18], however, the resulting low frequency data is not directly applicable to HEV use due to the dynamic nature of driving patterns and the extremely long time constant involved in the measurements. Furthermore, impedance techniques to model parameter quantities for a $36 \mathrm{~V}$ mild hybrid battery, is discussed in [19], and the impedance measurement of small to medium sized lead acid cells in [20].

Using a polar plot for a range of applied frequencies, an ideal capacitor and resistor in parallel will produce semicircular characteristics, with a diameter in the real axis equal to the resistance $R_{t}$, and radius in the imaginary axis corresponding to a value of $C_{\text {surface }}$ calculated from (1) [15].

$C_{\text {surface }}=\frac{1}{2 \pi f X}$

This leads to the standard method for deriving the value of the double layer capacitance, employing the value of the complex impedance at the peak of the capacitive semicircular arc. The high frequency intercept of the semicircle with the real axis corresponds to the value of $R_{i}$, whilst the low frequency intercept corresponds to the sum of $R_{i}$ and $R_{t}$.

However, the capacitive arc is often not well defined within a real cell, as demonstrated by the characteristics of the double terminal cell in Fig. 5, making it difficult to determine the magnitude of the double layer capacitance directly. This is due to the capacitance having contributions from both positive and negative electrodes; therefore the arc is rarely semicircular due to the dispersed and non-homogeneous properties of the cell plates. Hence, in practice the calculation of a value for $C_{\text {surface }}$ is often not straightforward.

Current interrupt techniques have also been used by some investigators for internal resistance measurement, typically utilising a relatively few points throughout the charge/discharge profile [21]. Others have shown that the electrical noise generated at the terminals of an SLI (Starting Lighting Ignition) battery during engine cranking may be processed to give internal resistance measurements, and hence, assess the SoC and SoH of the battery [22]. This approach may prove useful in HEV batteries, although, as is

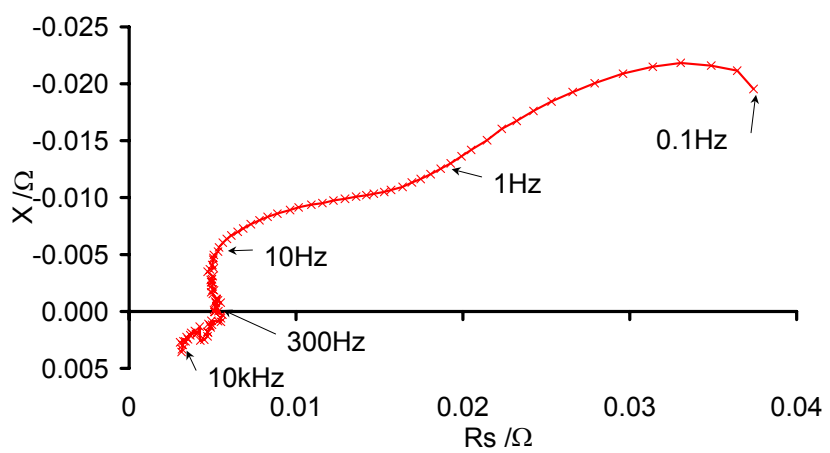

Fig. 5. Polar plot for a double terminal cell at $100 \%$ SoC. 


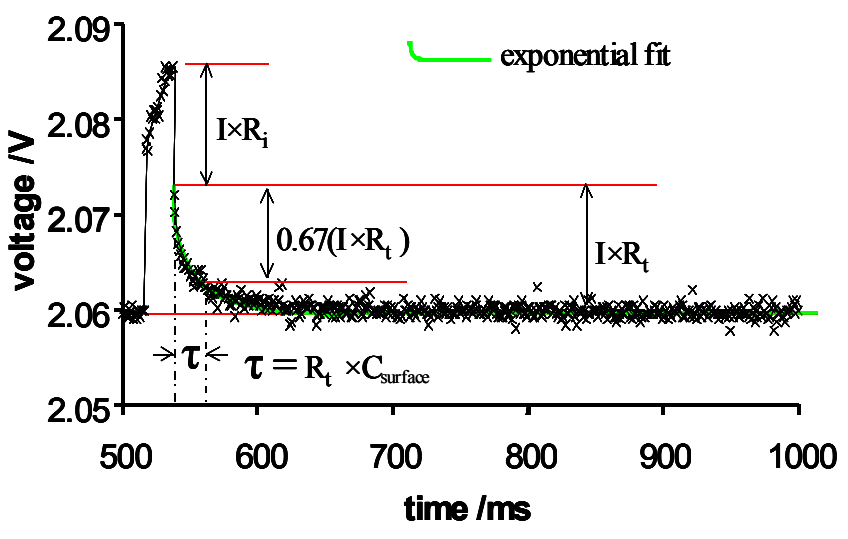

Fig. 6. Method of calculation of model parameters.

illustrated in Fig.7, the variation in $R_{i}$ and $R_{t}$ over much of the cell's SoC is relatively small, implying that resistance measurement can only usefully be used to detect low SoC.

The variation of the voltage and current response of the cell, with $\mathrm{SoC}$, is explored using a current interrupt technique during discharge. A short $10 \mathrm{~ms}$ interrupt is used to minimise the effect on the underlying discharge rate, whilst a relatively high number of measurements are made throughout the discharge. The cells are discharged from a $100 \%$ SoC using a constant current of 8A. During the discharge, $10 \mathrm{~ms}$ interruptions are periodically applied to the constant current, to facilitate the calculation of internal resistance. During each interrupt the cell voltage and current are sampled at $1 \mathrm{kHz}$, to exclude the effects due to low frequency polarisation. This high sampling rate data is then processed offline following each test to allow calculation of the cell's parameters. Figure 6 , shows an example of the voltage perturbation occurring during a current interrupt pulse.

Prior to testing, the cells are cycled on four full charge/discharge cycles, to bring them to a full and stable capacity. The discharge tests are then carried out at current level of 8A. Between each test, the cells are subjected to a full 16 -hour $10 \mathrm{~A}$ current limited, $2.45 \mathrm{~V}$ constant voltage charge at $25^{\circ} \mathrm{C}$.

A Labview routine is created to allow rapid offline calculation of the internal resistance for each of these interruptions. An automated routine is employed to process the data for 250 pulses applied during each discharge run to identify the parameters that fit the model, Fig. 4, according to the voltage and current changes seen on the trailing edge of each current interrupt pulse, as shown in Fig. 6.

On application of a transient signal, $C_{\text {surface }}$ will initially appear as a short circuit, and hence, the value of $R_{i}$ may be calculated from the initial voltage drop. The discharge of the double layer capacitor produces an exponential fall in voltage, until the voltage across the parallel combination of $C_{\text {surface }}$ and $R_{t}$ is equal to $I R_{t}$, the steady state voltage drop across $R_{t}$. An exponential fit is made to the portion of data following the initial IR drop to determine the time constant, $\mathrm{RC}$, of the parallel combination, and hence, $C_{\text {surface }}$. The results for the

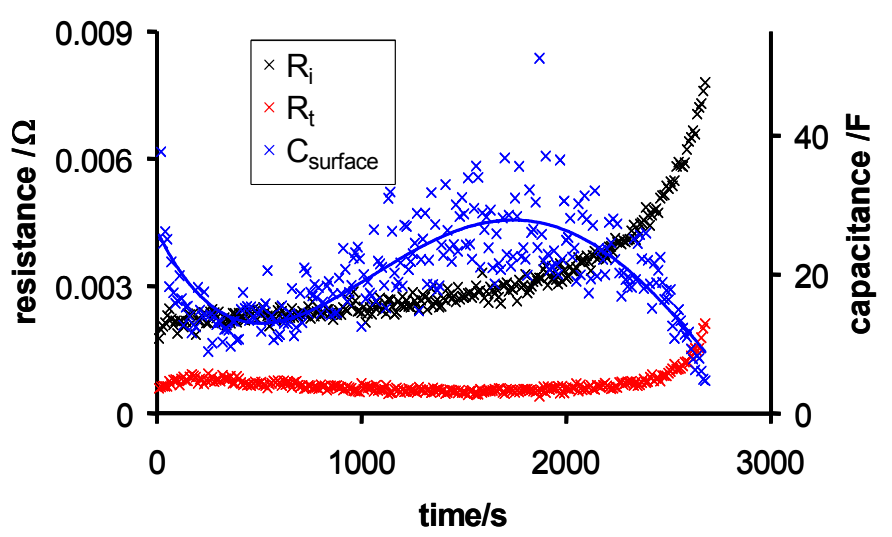

Fig. 7. Variation of model parameters over an $8 \mathrm{~A}$ discharge

test are plotted in Fig. 7, where $\mathrm{R}_{\mathrm{i}}$ rises slowly throughout the discharge until approximately $20 \% \mathrm{SoC}$ is attained, and then incurs a sharp rise, whilst the $R_{t}$ remains relatively constant throughout most of the discharge period, and rises abruptly at the end. There is an underlying trend that the surface capacitor value falls rapidly at the onset of discharge, then rising to maxima, prior to falling again at the end of discharge. The complete cell parameters are as follows:

TABLE I

DERIVED PARAMATERS FOR LEAD ACID CELL MODEL

\begin{tabular}{ll}
\hline \hline $\mathrm{C}_{\text {bulk }}$ & $88372 \mathrm{~F}$ \\
$\mathrm{C}_{\text {surface }}$ & $23 \mathrm{~F}$ \\
$\mathrm{R}_{\mathrm{i}}$ & $0.0026 \Omega$ \\
$\mathrm{R}_{\mathrm{t}}$ & $0.0005 \Omega$ \\
$\mathrm{R}_{\mathrm{d}}$ & $10000 \Omega$ \\
\hline \hline
\end{tabular}

\section{STATE VARIABLE DESCRIPTION OF BATTERY MODEL}

\section{A. State variables $V_{C b}, V_{C s}$ and $V_{0}$}

Voltages and currents describing the characteristics of the network shown in Fig. 4, are given by (note: by convention, current flowing into the cell is considered positive):

$V_{0}=I R_{i}+I_{1} R_{t}+V_{C b}$
$V_{0}=I R_{i}+V_{C s}+V_{C b}$
$V_{0}=I R_{i}+I_{1} R_{t}+I_{3} R_{d}$
$V_{0}=I R_{i}+V_{C s}+I_{3} R_{d}$

Moreover, taking the time-derivative of the output voltage, (2), and assuming $d I / d t \approx 0$ (the rate of change of terminal current, per sampling interval when implemented digitally, is negligible) gives the complete state variable description (5).

Furthermore, observability is satisfied under the mild conditions that $\quad C_{\text {bulk }} R_{d} \neq C_{\text {surface }} R_{t}, \quad C_{\text {bulk }} R_{d} \neq 1$ and $C_{\text {surface }} R_{t} \neq 1$. 


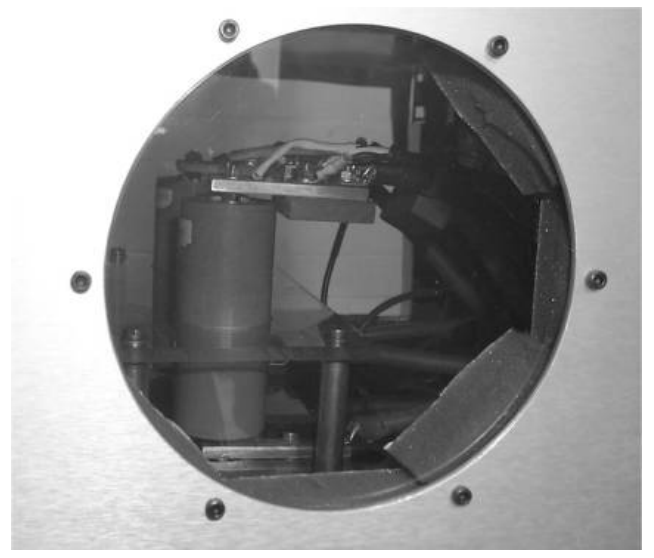

(a)

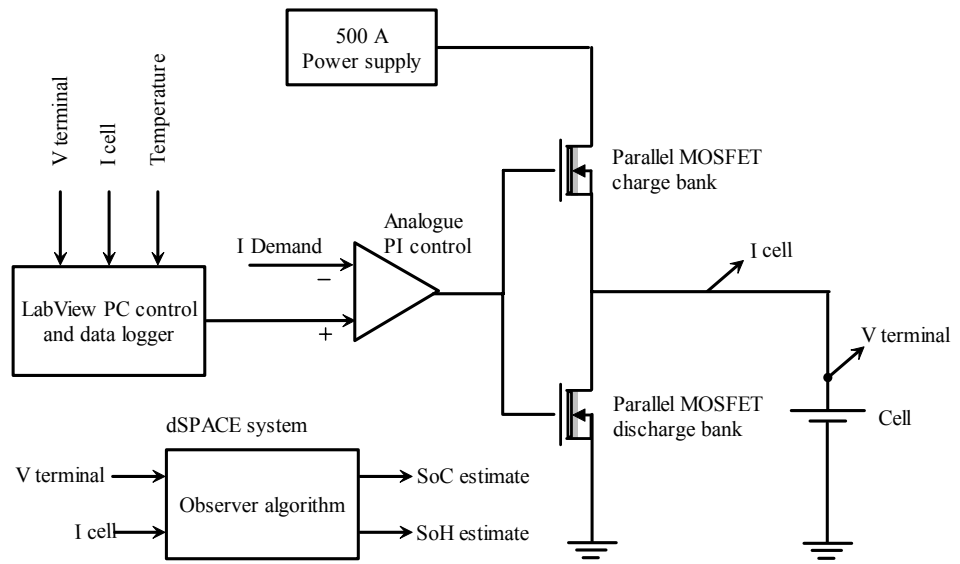

(b)

Fig. 8. (a) Picture of cell contained within a thermally controlled environmental chamber (b) High power cell test bench schematic.

$$
\left[\begin{array}{c}
\dot{V}_{C b} \\
\dot{V}_{C s} \\
\dot{V}_{0}
\end{array}\right]=\left[\begin{array}{ccc}
-\frac{1}{C_{\text {bulk }} R_{d}} & 0 & 0 \\
0 & -\frac{1}{C_{\text {surface }} R_{t}} & 0 \\
-\frac{1}{C_{\text {bulk }} R_{d}} & -\frac{1}{C_{\text {surface }} R_{t}} & 0
\end{array}\right] \cdot\left[\begin{array}{c}
V_{C b} \\
V_{C s} \\
V_{0}
\end{array}\right]+\left[\begin{array}{c}
\frac{1}{C_{\text {bulk }}} \\
\frac{1}{C_{\text {surface }}} \\
\frac{1}{C_{\text {bulk }}}+\frac{1}{C_{\text {surface }}}
\end{array}\right] I
$$

\section{VALIDATION OF LEAD ACID BATTERY MODEL}

\section{A. High power cell test bench}

A high-power cell test-bench has been constructed to allow continuous cycling of individual cells using custom powerand current- profiles, thereby permitting the characterisation of individual cells over closely managed operating duties. The cells are contained within a thermally controlled environmental chamber to provide known environmental conditions, Fig. 8(a).

Power for cell charging is supplied from a rectified $3 \phi$, $500 \mathrm{~A}$ power supply via a smoothing capacitor bank. The charge/discharge circuits employ an analogue PI controller driving two parallel arrays of MOSFET power devices, acting as variable resistors. One array of MOSFETs controls charging current to the cell, and the other, the discharge current, and both are forced air-cooled. The demand signal is provided via a PC-based Labview hardware development platform. Furthermore, a dSPACE system is employed in parallel with the LABview hardware. Measurements of the cell voltages and currents are sampled by 16-bit ADCs, from which the observer algorithms estimate SoC (and ultimately, $\mathrm{SoH})$. Figure 8(b) shows a schematic of one channel of the test bench.

\section{B. Behaviour of lead acid battery model}

Prior to the design of the observers, the validity of the proposed model is undertaken by subjecting both the cell (shown by Fig. 3) and the model, Fig. 4, to a discharge pulse of $1.53 \mathrm{~A}$, for a comparison of the modelled voltage to the measured cell voltage, Fig. 9. In addition, the behaviour of the bulk- and surface-capacitor voltages is also shown. Although there exists some discrepancy between the measured and modelled voltages, the underlying dynamic characteristics are essentially the same, with the principle difference being the voltage response between discharge pulses. The offset and drift are due to errors in initial condition estimates, and the effect of employing the model in an open-loop state over a prolonged period.

\section{Kalman Filter (KF) FOR SOC Estimation}

A discrete-time equivalent model of the system (5), can be obtained by assuming the applied input, $u$, is constant during each sampling interval [14]:

$x_{k+1}=\mathbf{A}_{\mathbf{d}} x_{k}+\mathbf{B}_{\mathbf{d}} u_{k}$

$y_{k+1}=\mathbf{C}_{\mathbf{d}} x_{k+1}$

where

$$
\mathbf{A}_{\mathbf{d}}=\left[\begin{array}{ccc}
1-\frac{T_{c}}{C_{\text {bulk }} R_{d}} & 0 & 0 \\
0 & 1-\frac{T_{c}}{C_{\text {surface }} R_{t}} & 0 \\
-\frac{1}{C_{\text {bulk }} R_{d}} & -\frac{1}{C_{\text {surface }} R_{t}} & 1
\end{array}\right], \quad \mathbf{B}_{\mathbf{d}}=\left[\begin{array}{c}
\frac{T_{c}}{C_{\text {bulk }}} \\
\frac{T_{c}}{C_{\text {surface }}} \\
\frac{T_{c}}{C_{\text {bulk }}}+\frac{T_{c}}{C_{\text {surface }}}
\end{array}\right],
$$

$\mathbf{C}_{\mathbf{d}}=\mathbf{C}$

and $\mathrm{T}_{\mathrm{c}}$ is the sampling period. For notational purposes, $\hat{x}_{(i / j)}$ represents an estimate of $x$ at step ' $i$ ' based on all the information up to, and including, time step ' $j$ '.

The stochastic principles underpinning the KF are appealing for this investigation, since it is recognised that the presence of disturbances stemming from sensor noise on the cell terminal measurements, and the use of non-ideal dynamic models, make it impossible to predict with certainty the states of the system over prolonged time periods - a statistical predictor/corrector formulation thereby provides obvious advantages. 

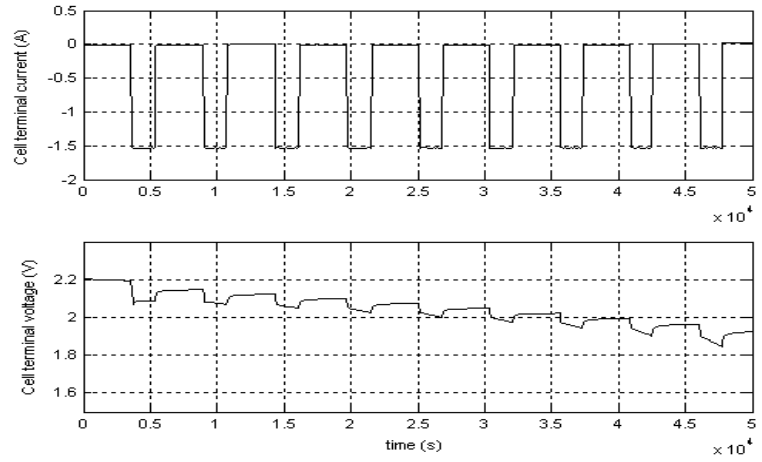

(a)

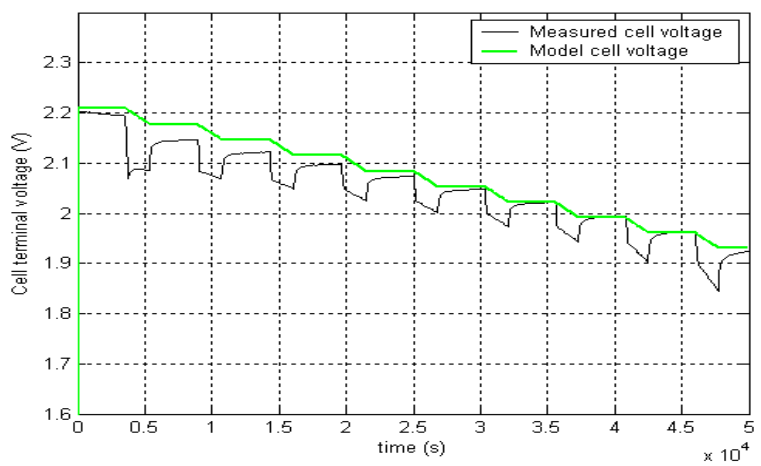

(b)
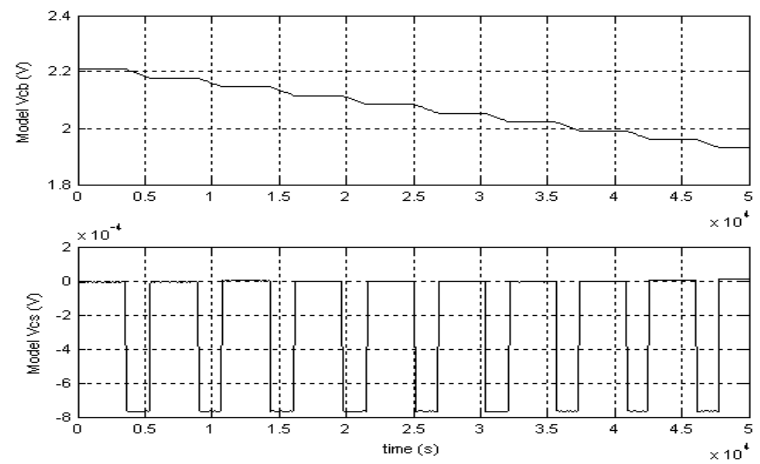

(c)

Fig. 9. Dynamic behaviour of lead acid battery model in response to discharge pulses of 1.53A (a) current and voltage of cell (b) measured and modelled cell voltage (c) modelled voltages across bulk and surface capacitors.

Since only terminal quantities of the battery can be measured, the input is defined as $u=I$, and the measured output is $y=V_{0}$. The predictor/corrector stages of the recursive KF algorithm applied is discussed in [14].

Although no formal stability and tuning methods are available for initialising the $\mathrm{KF}$, and recourse to empirical tuning is normally required, its use is nevertheless widespread. Information about the system noise contribution is contained in matrices $\mathbf{Q}$ and $\mathbf{R}$, and in essence, the selection of $\mathbf{Q}$ and $\mathbf{R}$ determines the accuracy of the filter's performance, since they mutually determine the action of the KF gain matrix, $\mathbf{K}_{k+1}$, and estimation error covariance matrix, $\mathbf{P}_{k+1 / k+1},[14]$. The covariance matrix representing measurement noise, $\mathbf{R}$, can be estimated from knowledge of the battery terminal voltage.
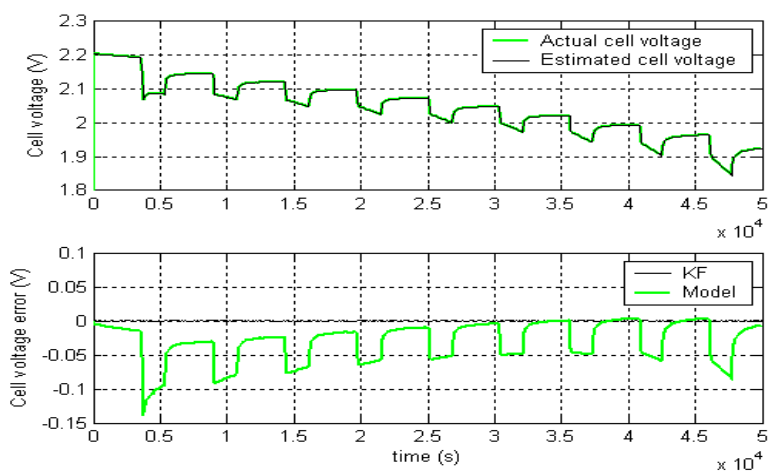

(a)
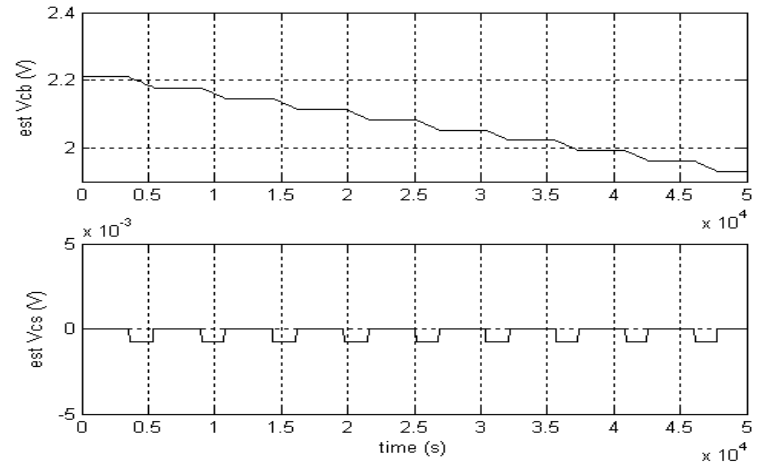

(b)

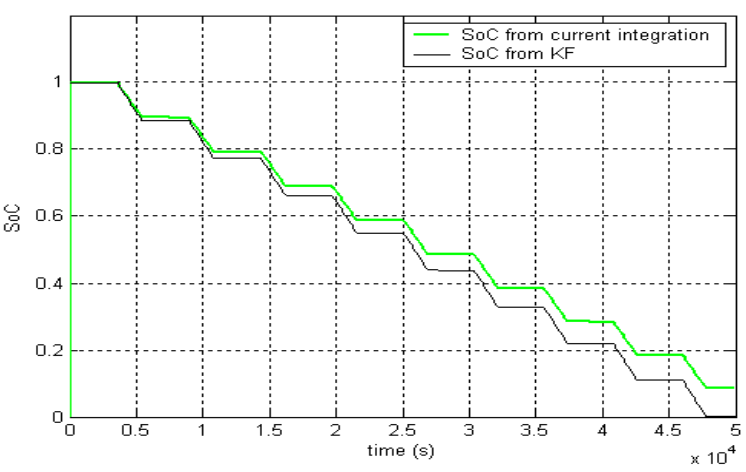

(c)

Fig. 10. Dynamic behaviour of KF estimator to discharge pulses of $1.53 \mathrm{~A}$ (a) actual and estimated cell voltage and voltage error from KF and cell model (b) estimated bulk and surface capacitor voltages (c) comparison of predicted SoC by method of current integration and KF respectively.

The variance is obtained from the square of the RMS noise on each cell, and is assumed to be Gaussian distributed and independent.

Initialisation of the covariance matrix describing disturbances on the plant, $\mathbf{Q}$, is complicated by the fact that knowledge of model inaccuracies and system disturbances is limited, particularly as each cell has different characteristics. A judicious choice of $\mathbf{Q}$ is therefore obtained from experimental studies under the simplifying assumption that there is no correlation between the elements of $\sigma_{k}$ and the noise present on each cells voltage transducer, thereby leading to a diagonal $\mathbf{Q}$. The initial covariance matrix, $\mathbf{P}_{\mathbf{0}}$, together with $\mathbf{Q}$ and $\mathbf{R}$ are ultimately chosen to be: 


$$
\mathbf{P}_{\mathbf{0}}=\left[\begin{array}{lll}
1 & 0 & 0 \\
0 & 1 & 0 \\
0 & 0 & 1
\end{array}\right], \mathbf{Q}=\left[\begin{array}{ccc}
1.549 \times 10^{-5} & 0 & 0 \\
0 & 0.4191 & 0 \\
0 & 0 & 0.8143
\end{array}\right], \mathbf{R}=10
$$

\section{A. Practical Implementation of the Kalman Filter}

The benefits of employing an observer-based technique, as opposed to the battery model (5), are now shown. As described previously (Sect. IV-B) a discharge pulse of 1.53A is applied to both the experimental test battery and to the realtime KF, with feedback of terminal current as its input. A comparison of the actual and estimated output cell voltage, and the error associated in the calculation of voltage using a model-based technique, compared to an observer, is shown in Fig. 10(a). In particular, the KF shows excellent convergence of the states, and the output voltage, with negligible error.

The voltages across the bulk and surface capacitors, obtained from the KF, is shown in Fig. 10(b). The discharge pulses are applied until the cell is completely discharged, where the $\mathrm{SoC}$ as predicted by the current integration method incurs a large error. However, the KF demonstrates improved performance by accurately estimating the $\mathrm{SoC}=0$ at this point, Fig. 10 (c). Note that $\mathrm{SoC}=1$ is a normalized value used to define a fully-charged cell and is proportional to the bulk capacitor voltage.

It is notable that, for linear systems, after several iterations, $\mathbf{K}_{k+1}$ converges to a constant matrix, and, in such cases $\mathbf{K}_{k+1}$ can be pre-calculated off-line, thereby presenting significant savings in computational load at the expense of potentially incurring a loss of accuracy during transients [14].

The impact of the covariance matrix $\mathbf{R}$ on the estimation performance of the KF, has been previously considered [14] where it is seen that although significant noise is present on the cell terminal voltage measurements, careful selection of $\mathbf{R}$ can provide estimates with enhanced noise immunity.

\section{B. Comparison of SoC Estimation Techniques}

The KF is now applied to a driving cycle (RHOLAB, Fig. 2) from which, the real-time estimation of $\mathrm{SoC}$ of a single cell is determined. Prior to the RHOLAB driving cycle the cell is discharged to $\mathrm{SoC} \approx 0.8$, this being the defined operating point for Partial State-of-Charge (PSoC) on the HEV driving profile; with the current profile is shown by Fig. 11(a).

Figure 11(b) shows a comparison of the actual- and estimated- output voltage, and the negligible voltage error from the use of the KF. The voltages across the bulk and surface capacitors are shown in Fig. 11(c). Furthermore, Fig. 11(d) shows results using conventional SoC estimation, by the integration of current method, with charging efficiency of 0.97 [1], and those from the proposed KF scheme. Having been subjected to a RHOLAB driving cycle, at $\mathrm{t}=1400$ s (time from start of the RHOLAB profile) the tests are terminated, and the remnant charge in the cell is measured using a $1.53 \mathrm{~A}$ discharge, to a terminal voltage of $1.7 \mathrm{~V}$ and noting the remaining Ampere-hours, Fig. 12, $\left(\begin{array}{lll}1.87 & \mathrm{Ah}\end{array}\right)$. This corresponds to a final SoC of the cell of 0.283 . From Fig. 12,

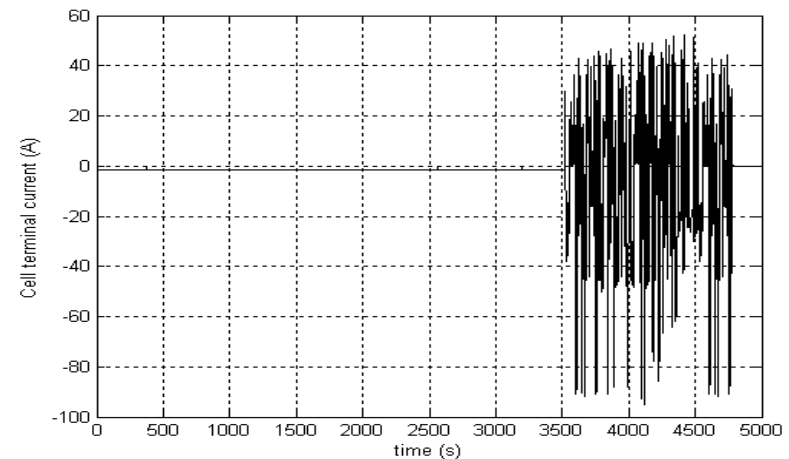

(a)
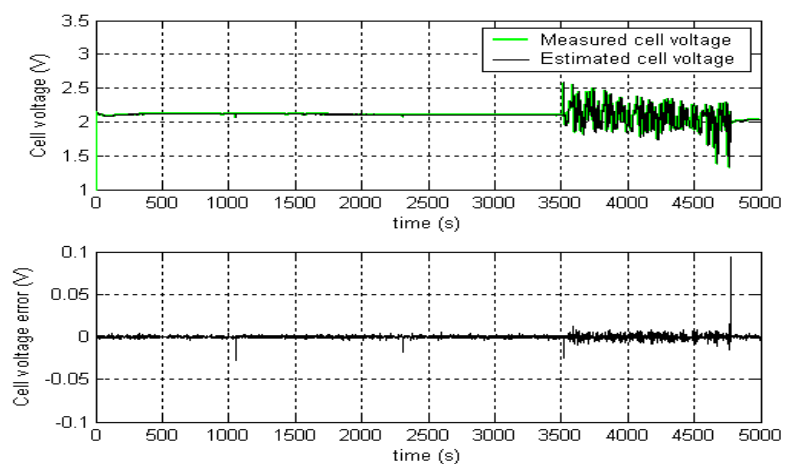

(b)
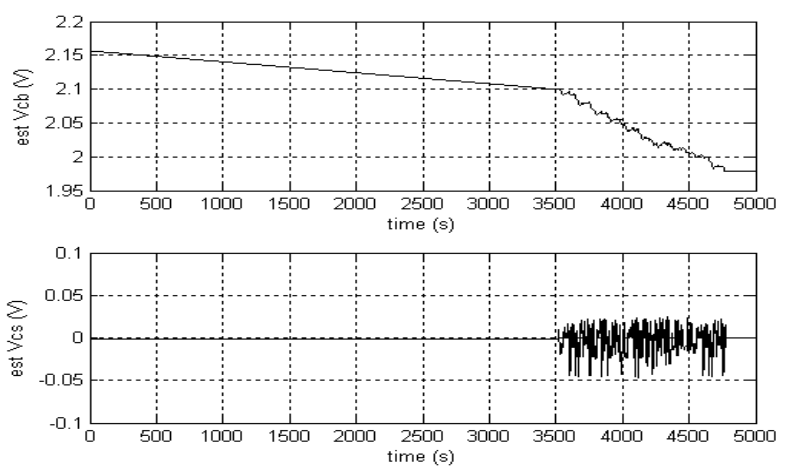

(c)

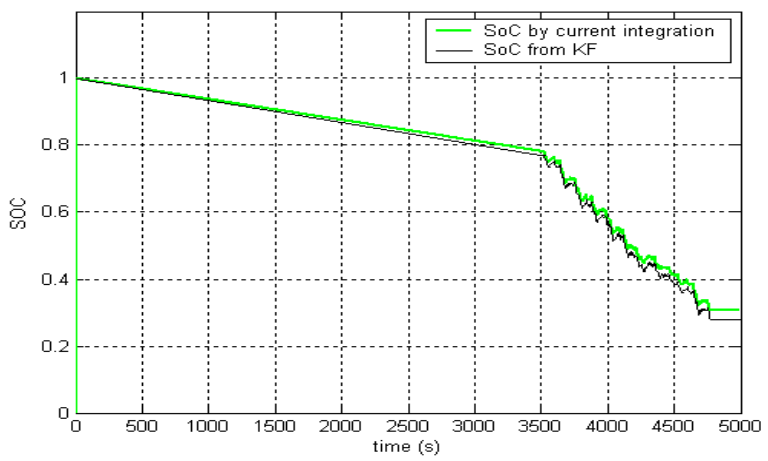

(d)

Fig. 11. Implementation of KF on RHOLAB cycle (a) Discharging a cell from SoC of 1 to 0.8 using 1.53A discharge subsequent to a RHOLAB driving profile as shown by Fig 2 (b) measured and estimated cell voltage and cell voltage error (c) estimated bulk and surface capacitor voltage (d) comparison of SoC determined by conventional means (integration of current) and from estimated bulk capacitor voltage. 
and Table II, it can be seen that the performance of the KF is excellent, and, although both $\mathrm{SoC}$ estimation techniques follow the correct profile, the integration-based method demonstrates significant drift over time, with an error of $\approx 4 \%$ ensuing, whereas the KF consistently provides estimates within $\approx 1 \%$ of the measured values.

\section{State of Health estimation}

SoH is the ability of a cell to store energy, source and sink high currents and retain charge over extended periods, relative to its initial or nominal capabilities. The available charge stored within a fully-charged cell is expected to fall with cell usage, as active material on the cell plates gradually degrades by mechanisms such as, loss of plate active surface area due to repeated dissolution and re-crystallisation, loss of electrical contact between metallic grids and active materials, and growth of large inactive crystals of lead sulphate. Such capacity-loss mechanisms generally occur slowly in VRLA batteries that are cycled at low-rates over their full SoC range. However, when operated as a peak power buffer, in a HEV system, the cells are operated at a PSoC i.e. the cell is never cycled over its full SoC range, and is subjected to both high charge and discharge currents. Studies have shown that this PSoC operation can lead to truncated service life in VRLA cells due to build-up of sulphate within the plate structures, as a result of inefficient recharge of plate material $[23,24]$. Such capacity loss can be deemed a loss of cell SoH. Early detection of SoH degradation would allow a 'smart' battery pack to take remedial action, such as the application of conditioning routines to the cell, to remove small sulphate crystals before they form large inactive crystals, thereby restoring the cells capacity. Measuring cell capacity by the standard means of a low current discharge is impractical for HEV applications, and online techniques that utilise only cell terminal measurements, made whilst the HEV is driven, are therefore required.

\section{A. EKF-based Estimation of $\mathrm{SoH}$}

A means of estimating bulk capacitance $C_{b u l k}$, requires adding an extra state, $d C_{b u l k} / d t=0$, into the observer structure (10) and assuming the rate-of-change of $C_{b u l k}$, over a sampling interval, is negligible. Since the derivatives of $V_{C b}$ and $V_{0}$ are coupled by non-linear elements, an EKF is now required for effective estimation of state variables. It should be noted, however, that the realisation results in an increase in order of the resulting Jacobians, covariance, noise and disturbance matrices, with a consequential increase in computation overhead. The proposed non-linear battery model is written in the form:

$$
\begin{aligned}
& \dot{x}=f(x, u) \\
& y=C(x)
\end{aligned}
$$

where $f(x, u)$ is given by $(10)$.

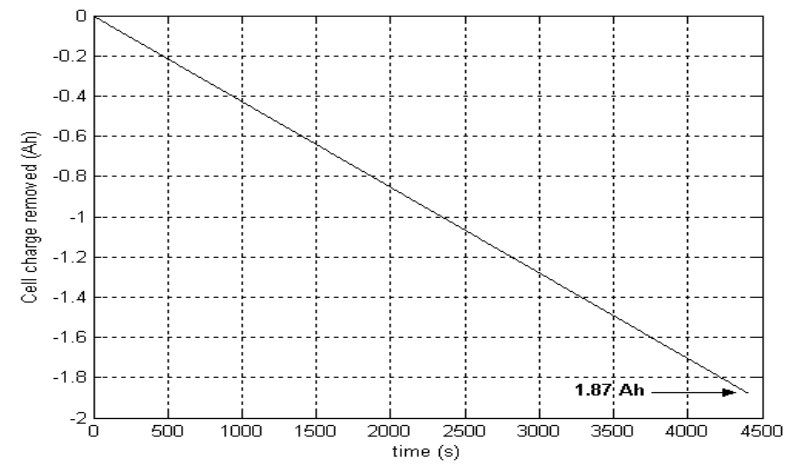

Fig. 12. Cell discharge characteristic to $0 \%$ SoC.

TABLE II

COMPARISON OF STATE OF CHARGE ESTIMATION FROM CURRENT INTEGRATION METHODAND KF

\begin{tabular}{ll}
\hline \hline & Final SoC estimation \\
Measured SoC & 0.283 \\
Current integration SoC & 0.3177 \\
Kalman Filter SoC & 0.2793 \\
\hline \hline
\end{tabular}

$$
x=\left[V_{C b}, V_{C s}, V_{0}, \alpha\right]^{T},\left[\begin{array}{c}
-\frac{V_{C b} \alpha}{R_{d}}+I \alpha \\
-\frac{V_{C s}}{C_{\text {surface }} R_{t}}+\frac{I}{C_{\text {surface }}} \\
-\frac{V_{C b} \alpha}{R_{d}}-\frac{V_{C s}}{C_{\text {surface }} R_{t}}+\left(\alpha+\frac{1}{C_{\text {surface }}}\right) \\
0
\end{array}\right],
$$

and $C(x)=V_{0}, \alpha=1 / C_{\text {bulk }}$.

The EKF requires a small-signal model of the system, at each sample step, by linearising (10) about the current operating point, $x_{0}, u_{0}$. From a Taylor series expansion, (ignoring the presence of noise):

$\delta \dot{x}=\mathbf{F}(x) \delta x+\mathbf{B} \delta u, \quad \delta y=\mathbf{C} \delta x$

where,

$\delta x=x-x_{0} \quad \delta u=u-u_{0}$

$\mathbf{F}(x)=\left.\frac{\partial f}{\partial x}\right|_{x(t), u(t)}=\left[\begin{array}{cccc}-\frac{\alpha}{R_{d}} & 0 & 0 & -\frac{V_{C b}}{R_{d}}+I \\ o & -\frac{1}{C_{\text {surface }} R_{t}} & 0 & 0 \\ -\frac{\alpha}{R_{d}} & -\frac{1}{C_{\text {surface }} R_{t}} & 0 & -\frac{V_{C b}}{R_{d}}+I \\ 0 & 0 & 0 & 0\end{array}\right]$

$\mathbf{B}=\left.\frac{\partial f}{\partial u}\right|_{x(t), u(t)}=\left[\begin{array}{llll}\alpha & \frac{1}{C_{\text {surface }}} & \alpha+\frac{1}{C_{\text {surface }}} & 0\end{array}\right]^{T}$

$\mathbf{C}=\left.\frac{\partial C}{\partial x}\right|_{x(t), u(t)}=\left[\begin{array}{llll}0 & 0 & 1 & 0\end{array}\right]$ 


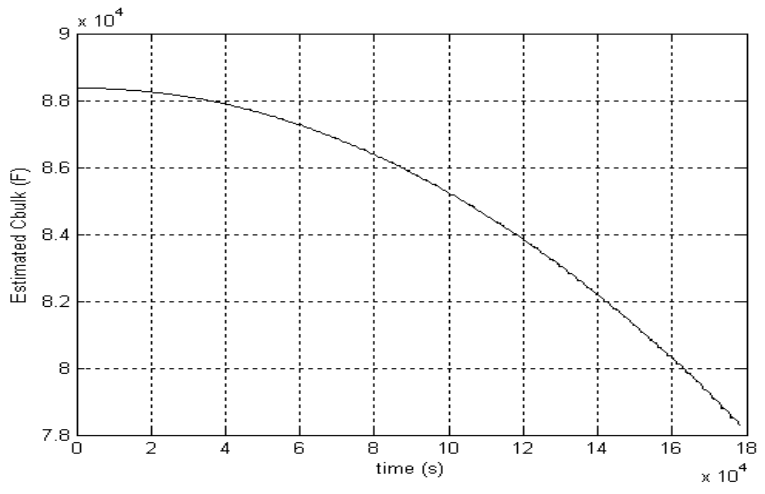

(a)

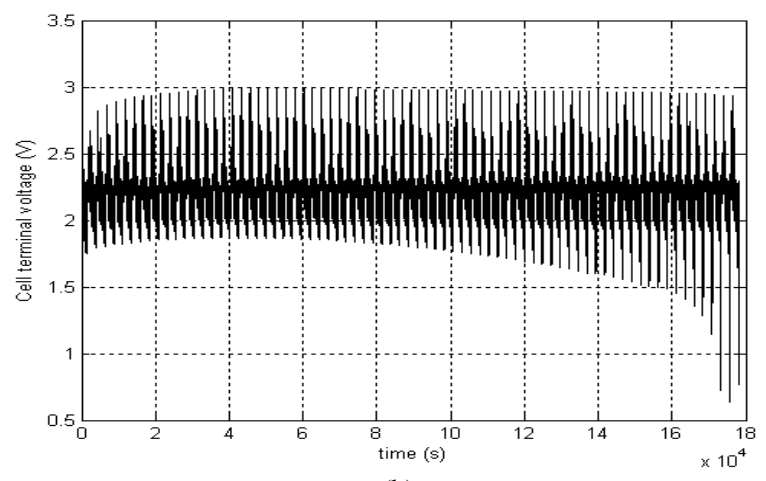

(b)

Fig. 13. EKF employed to predict SoH of cell pack (a) estimated bulk capacitance $C_{\text {bulk }}(\mathrm{b})$ cell terminal voltage

The resulting small signal model, about the operating point $x_{0}, u_{0}$, is given by:

$\delta \dot{x}=\mathbf{A}_{k} \delta x+\mathbf{B}_{k} \delta u, \quad \delta y=\mathbf{C} \delta x$

(where $\mathbf{A}_{k}=\left.\mathbf{F}(x)\right|_{x_{k}, u_{k}}$ ) that can be discretized, to give:

$x_{k+1}=\mathbf{A}_{\mathbf{d}} x_{k}+\mathbf{B}_{\mathbf{d}} u_{k} \quad y_{k+1}=\mathbf{C}_{\mathbf{d}} x_{k+1}$

and used in the KF algorithm described previously in [14].

\section{B. Online implementation of $\mathrm{SoH}$ monitoring}

The SoH of known cells has previously been evaluated [23] by performing continuous power cycling tests (using the RHOLAB profile shown in Fig. 2, on two separate battery packs, each consisting of 18 cells. Results of the tests have demonstrated similar pack lifetimes in each case, over 77 and 74 power cycles, respectively. During the tests, the cells of both packs suffered a measurable mean loss in cell capacity of $\approx 0.01 \mathrm{Ah}$, per power cycle [23]. Whilst performing the tests, the EKF is employed, and the variation of $C_{b u l k}$ is estimated. The results, shown in Fig. 13(a), demonstrate the ability of the state observer scheme to estimate $C_{b u l k}$, over time, ultimately indicating a reduction of $\approx 10 \%$ during the tests, corresponding to an average loss of cell capacity of $0.8 \mathrm{Ah}$ for a nominal $8 \mathrm{Ah}$ cell. This, therefore, compares well to the measured loss of capacity of $\approx 0.77 \mathrm{Ah}$ from the packs under test. Figure $13(\mathrm{~b})$ shows the applied cell terminal voltage profile.

An additional test to determine $\mathrm{SoH}$ is performed on a newly conditioned Hawker cell, Fig. 3. Subsequent to the conditioning routine, the cell undergoes a series of operational profiles, as shown in Fig. 14. It should be noted that the term

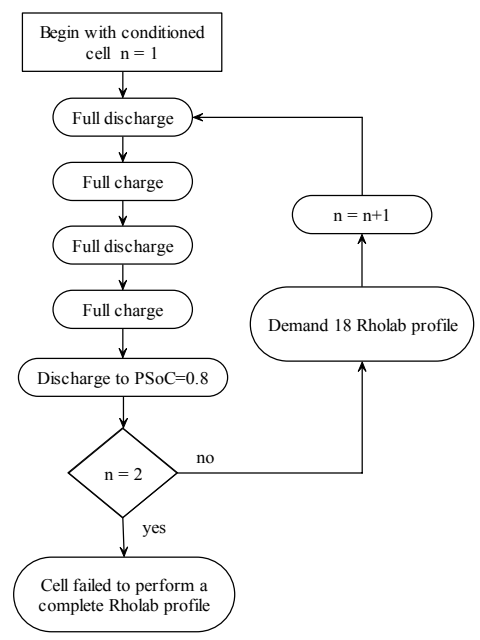

Fig. 14. Profile subject to a RHOLAB cell
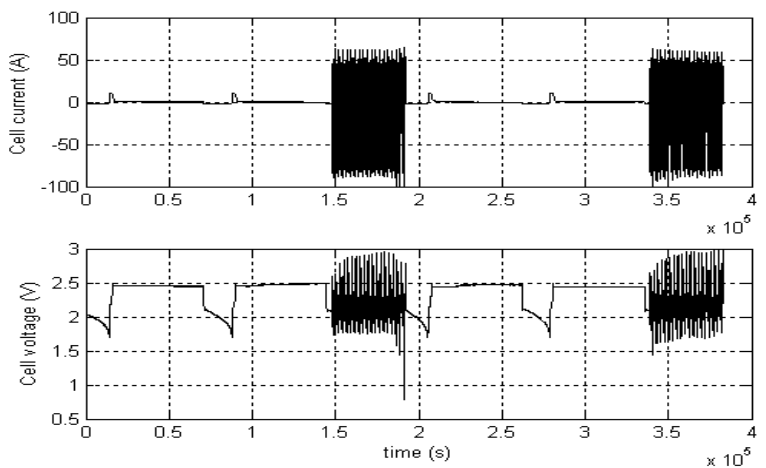

(a)

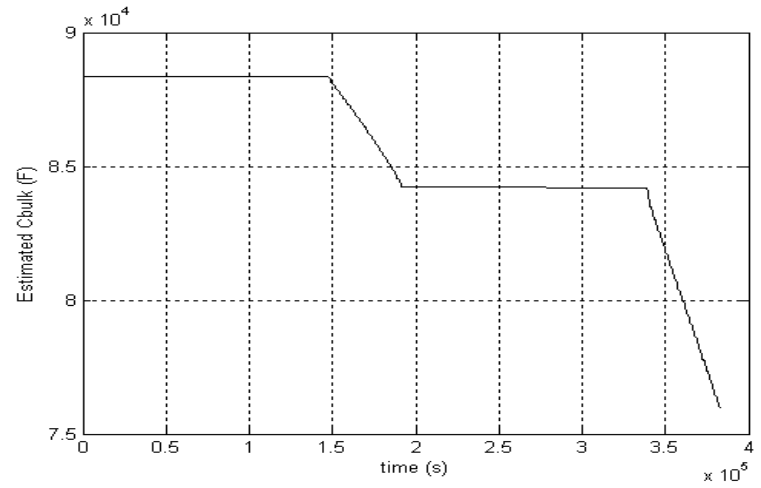

(b)

Fig. 15. EKF employed to predict $\mathrm{SoH}$ of cell pack (a) cell terminal current and voltage (b) estimated bulk capacitance $C_{b u l k}$

"full discharge" is used to signify a complete discharge to $1.7 \mathrm{~V}$ at rate of $1.53 \mathrm{~A}$, and "full charge" describes charging for 16 hours at $2.45 \mathrm{~V}$ with a $10 \mathrm{~A}$ current limit.

Figure 15(a) shows the cell terminal current and voltage profile. Notably, from Fig. 15(b) it is seen that the $\mathrm{SoH}$ is impervious to the full charge/discharge routines applied to the cell, with negligible degradation in SoH occurring. However, on demanding 18 repetitive RHOLAB profiles, Fig. 2, the $\mathrm{SoH}$ is seen to rapidly decline, Fig. 15(b). Furthermore, from a comparison with results from Fig 13, it can be seen that the cell fails to perform an additional complete RHOLAB profile, due to cell capacity loss of $\approx 10 \%$. 


\section{CONCLUSION}

The paper presents an alternative approach for estimating the $\mathrm{SoC}$ and $\mathrm{SoH}$ of a cell pack using KF, and EKFs, respectively, by modelling the dynamic behaviour of each cell based on a generic Randle's equivalent circuit comprising of two-capacitors (bulk and surface) and three resistors, (terminal, transfer and self-discharging). Although it is shown that the model is not an accurate representation of the cell model, rather than increasing the complexity of the cell model to closely match the real system, the application of a KF, with its inherent predictor-corrector mechanism, is shown to be robust to such modelling deficiencies. In particular, a comparison of SoC estimates using the presented $\mathrm{KF}$ technique, and the more conventional coulomb-counting techniques, is undertaken using "road data" collected from a Honda Insight HEV driven on a test track. The presented results show a significant improvement in SoC estimation from the proposed KF methodology.

Furthermore, extensions for SoH monitoring, by employing an EKF, have also been presented-using only measurements of cell terminal quantities as input. Such data is extremely important for HEV manufacturers since the ability to analyze State-of-Function (SoF), which is dependent on both SoC and $\mathrm{SoH}$ information, is feasible. The SoF ultimately describes the ability of a cell to perform adequately under HEV demands, providing prediction of available capacity, and discharge and recharge capability, thereby allowing a 'smart' battery to forecast the response of the cell to driving demands, and leading to optimal utilisation of the battery pack with regard to performance and lifetime, and therefore, better overall energy management within the vehicle.

\section{ACKNOWLEDGMENT}

The authors wish to acknowledge the financial assistance provided by the UK's DTI Foresight Vehicle Link Program, and the EPSRC, who have funded the RHOLAB project jointly with the European Advanced Lead Acid Battery Consortium, and Hawker Batteries. They would also like to acknowledge the support given by the other partners in the project, namely, Provector Ltd, the University of Warwick and Hawker Batteries (now part of Enersys Inc.).

\section{REFERENCES}

[1] M. J. Kellaway, P. Jennings, D. Stone, E. Crowe, A. Cooper. (2003) "Early results from a systems approach to improving the performance and lifetime of lead acid batteries." Elsevier. Journal of Power Sources. Vol.116. pp. 110-117.

[2] S. Piller, M. Perrin, A. Jossen. (2001). "Methods for state-of-charge determination and their applications." Elsevier. Journal of Power Sources. Vol. 96. pp. 113-120.

[3] O. Caumont, P. Le Moigne, X. Muneret, P. Lenain, C. Rombaut. (1998). "An optimised state of charge algorithm for lead-acid batteries in electric vehicles." Electric Vehicle Symposium, EVS-15, 1998, CD-ROM.

[4] H. Bode. (1977). "Lead acid Batteries." Wiley. ISBN 0-471-08455-7.

[5] F. Huet. (1998). "A review of impedance measurements for determination of the state-of-charge or state of health of secondary batteries." Elsevier. Journal of Power Sources. Vol. 70. pp. 59-69.
[6] S. Rodrigues, N. Munichandraiah, A. K. Shukla. (2000). "A review of state-of-charge indication of batteries by means of a.c. impedance measurements." Elsevier. Journal of Power Sources. Vol.87. pp. 12-20.

[7] C. C. Chan, E.W.C. Lo, S. Weixiang. (2000). "The available capacity computation model based on artificial neural network for lead acid batteries in electric vehicles." Elsevier. Journal of Power Sources. Vol. 87. pp. 201-204.

[8] P. Singh, C. Fennie, D. Reisner, A. Salkind. (1998). "A fuzzy logic approach to state-of-charge determination in high performance batteries with applications to electric vehicles." Electric Vehicle Symposium 15. CD-ROM.

[9] G. O. Patillon J. N. d'Alché-Buc. (1997). "Neural network adaptive modelling of battery discharge behavior." Artificial Neural Networks ICANN'97 $7^{\text {th }}$ International Conference, Springer-Verlag, Berlin, Lecture notes in computerscience. Vol.1327. pp. 1095-1100.

[10] S. R. Bhatikar, R.L. Mahajan, K. Wipke, V. Johnson. (1999). "Neural network based energy storage system modelling for hybrid electric vehicles." National Renewable Energy Laboratories, August 1999.

[11] P. Mauracher, E. Karden. (1997). Dynamic modelling of lead/acid batteries using impedance spectroscopy for parameter identification. Elsevier. Journal of Power Sources. Vol. 67. pp. 69-84.

[12] R. E. Kalman, (1982) "A new approach to linear filtering and prediction problems." Transactions of the ASME - Journal of Basic Engineering, Series D, pp 35-45..

[13] R. Bucy, R. Kalman, I. Selin. (1965) "Comment on the Kalman filter and nonlinear estimates of multivariate normal processes." IEEE Transactions on Automatic Control, 10(1), pp.118-119.

[14] B. S. Bhangu, P. Bentley, D. A. Stone, C. M. Bingham. (2005) "Nonlinear Observers for Predicting State-of-Charge and State-of-Health of Lead-acid Batteries for Hybrid Electric Vehicles", IEEE Trans. Vehicular Technology Vol. 54, No. 3, pp. 783-794.

[15] D. Linden. (1995). Handbook of Batteries. McGraw-Hill inc. 2nd Ed. ISBN 0-07-037921-1.

[16] J. Ross Macdonald, W. R. Kenan. (1987). Impedance Spectroscopy: Emphasizing Solid Materials and Systems. Wiley. ISBN: 0-471-831220.

[17] E. Karden, S. Buller, R. W. De Donker. (2002). A frequency-domain approach to dynamical modelling of electrochemical power sources. Pergamon. Electrochimica Acta. Vol. 47. pages 2347-2356.

[18] P. Mauracher, E. Karden, K. Rembe. (1996). Measurement of the ultralow frequency impedance of lead acid batteries. Proceedings of LABAT '96. Varna, Bulgaria. pages 91-94.

[19] S. Buller, M. Thele, E. Karden, R. W. De Doncker. (2003). Impedancebased non-linear dynamic battery modeling for automotive applications. Elsevier. Journal of Power Sources. Vol.113. pages 422-430.

[20] A. Salkind, T. Atwater, P. Singh, S. Nelatury, S. Damodar, C. Fennie Jr., D. Reisner. (2001). Dynamic characterization of small lead-acid cells. Elsevier. Journal of Power Sources. Vol.96. pages 151-159.

[21] C. S. C. Bose, D. Williams, S. McCluer, M. J. Model. (2001). Lessons learned using Ohmic techniques for battery modelling. IEEE. $16^{\text {th }}$ annual battery conference on applications and advances. pages 99-104.

[22] S. Buller; J. Walter; E. Karden; R.W. De Doncker. (2002). ImpedanceBased Online Monitoring of Automotive Batteries. Advanced Automotive Battery Conference (AABC 02), $4^{\text {th }}-7^{\text {th }}$ February 2002. Las Vegas, USA.

[23] P. Bentley, D.A. Stone. (2004). Lifetime extension of Valve regulated lead acid (VRLA) batteries under hybrid vehicle duty. IEE. Proceedings of the $2^{\text {nd }}$ international power electronics machines and drives conference. PEMD2004. Edinburgh, Scotland. Vol. 1. pages 49-54.

[24] J. Jones. (1991, May 10). Networks (2nd ed.) [Online]. Available: Hollenkamp, Baldsing, Lim et al. "Overcoming negative-plate capacity loss in VRLA batteries cycled under partial state-of-charge duty," ALABC Investigation report ET/IR398R, 2001. 